\title{
The effective volumes of waters of crystallization: non-ionic pharmaceutical systems
}

\section{Leslie Glasser}

Acta Cryst. (2019). B75, 784-787

\section{IUCr Journals CRYSTALLOGRAPHY JOURNALS ONLINE}

Copyright (C) International Union of Crystallography

Author(s) of this article may load this reprint on their own web site or institutional repository provided that this cover page is retained. Republication of this article or its storage in electronic databases other than as specified above is not permitted without prior permission in writing from the IUCr.

For further information see http://journals.iucr.org/services/authorrights.html 


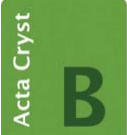

STRUCTURAL SCIENCE CRYSTAL ENGINEERING MATERIALS

ISSN 2052-5206

Received 29 May 2019

Accepted 22 July 2019

Edited by C. M. Reddy, IISER Kolkata, India

Keywords: crystal engineering; thermodynamics; pharmaceuticals; hydrates; anhydrates; volume; water of crystallization.

Supporting information: this article has supporting information at journals.iucr.org/b

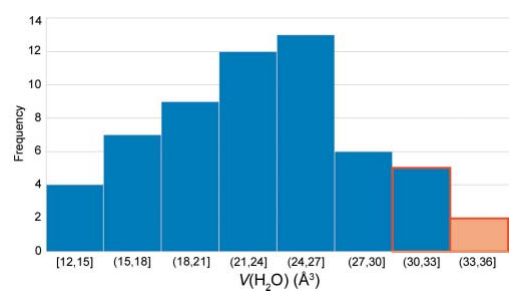

C 2019 International Union of Crystallography

\section{The effective volumes of waters of crystallization: non-ionic pharmaceutical systems}

\author{
Leslie Glasser* \\ Curtin Computation Group, Discipline of Chemistry, Curtin University, GPO Box U1987, Perth, West Australia 6845, \\ Australia. *Correspondence e-mail: I.glasser@curtin.edu.au
}

The physical properties of organic solids are altered when hydrated (and, more generally, when solvated) and this is of particular significance for pharmaceuticals in application; for instance, the solubility of a hydrate is less than that of its parent. The effective volumes of waters of crystallization for non-ionic pharmaceuticals (where the 'effective' volume is the difference per water molecule between the hydrate volume and the volume of the anhydrous parent) are here examined. This investigation contrasts with our earlier study of effective volumes of waters of crystallization for ionic materials where the coulombic forces are paramount. Volumetric properties are significant since they correlate strongly with many thermodynamic properties. Twenty-nine hydrate/parent systems have been identified, and their volumetric properties are reported and analysed (apart from aspartame and ephedrine for which the structural data are inconsistent). Among these systems, the data for paracetamol are extensive and it is possible to differentiate among the volumetric properties of its three polymorphs and to quantify the effect of temperature on their volumes. The effective volumes in both ionic and non-ionic systems are similar, with a median effective volume of $22.8 \AA^{3}$ for the non-ionic systems compared with $24.2 \AA^{3}$ for the ionic systems, and both are smaller than the molecular volume of $30 \AA^{3}$ of ambient liquid water - which appears to be an upper limit to the effective volumes of waters of crystallization under ambient conditions. These results will be supportive in checking and confirmation of hydrated crystal structures and in assessing their thermodynamic properties.

\section{Introduction}

Over the last few years, colleagues and I have studied the thermodynamics of solvation and, more specifically, of hydration (Glasser \& Jenkins, 2007; Glasser \& Jones, 2009; Glasser, 2014; Glasser, 2019), utilizing both our thermodynamic difference rules (TDR) (Jenkins \& Liebman, 2009) and our volume-based thermodynamics (VBT) methods (Glasser \& Jenkins, 2016). TDR provides difference relations between thermodynamic quantities, $P$ (where $P$ may represent quantities such as formula volume $V_{\mathrm{m}}$, entropy $S$, enthalpy $H$, Gibbs energy $G$, heat capacity $C_{\mathrm{p}}$ or $C_{\mathrm{v}}$, for example), for materials having the same basic chemical formula (Jenkins \& Glasser, 2004). Thus, for hydrates (and similarly for solvates in general)

$$
P\left(M_{p} X_{q} \cdot m \mathrm{H}_{2} \mathrm{O}\right)-P\left(M_{p} X_{q} \cdot n \mathrm{H}_{2} \mathrm{O}\right)=(m-n) \theta_{P, \mathrm{H} 2 \mathrm{O}},
$$

where $\theta_{P, \mathrm{H} 2 \mathrm{O}}$ represents a mean difference of $P$ among hydrates, and $m$ or $n$ may be zero. Such relations rely on an additivity among the thermodynamic quantities which is well attested in the reports referenced above. VBT provides a 
complementary set of relations describing correlations between the thermodynamic quantities, $P$, and formula volume, $V_{\mathrm{m}}$. For example, for entropy estimation,

$$
S_{\mathrm{est}}=\alpha V_{\mathrm{m}}+\beta,
$$

where $\alpha$ and $\beta$ are constants for a related group of materials such as ionic solids or hydrates or organic solids (Jenkins \& Glasser, 2003; Glasser \& Jenkins, 2004).

In order to analyse such relations beyond simple mean values, we have recently (Glasser, 2019) examined the variability of the effective volume of waters of crystallization in ionic systems (where the effective volume is the volume difference per hydration water between a hydrate and its parent anhydrous material). We have introduced the term 'effective' in order to recognize that there may be some rearrangement of the crystal structure of the parent on hydration in order to accommodate the introduced water, with a resulting small alteration in its formula volume, although the constituent atoms and the water molecule itself are generally regarded as incompressible (Hofmann, 2002). For convenient reference to the published crystallographic data, we use the secondary SI unit of $\AA^{3}$ for volume; to convert to the standard SI unit of $\mathrm{nm}^{3}$ used elsewhere in our studies, divide by 1000 .

We have observed that there is a firm upper bound of $30 \AA^{3}$ to the effective volume per water molecule in ionic systems, which corresponds closely to the volume per water molecule in liquid water under ambient conditions (and is less than the corresponding volume of $32 \AA^{3}$ of hexagonal ice, with its open hydrogen-bonded framework structure). A median effective volume of $24.2 \AA^{3}$ was found for ionic systems from 182 hydrate/parent pairs (Glasser, 2019).

In the current work, we examine the effective volume of water of crystallization in strictly non-ionic, organic pharmaceutical systems, deliberately excluding systems with charges in order to contrast with ionic systems where coulombic forces may dominate.

\section{Data collection}

In order to locate data sets for hydrate/parent pairs from among the $\sim 1000000$ organic structures reported by the Cambridge Structural Database (CSD) (Groom et al., 2016), we have been guided by the Hydrates Database (Fucke et al., 2019) which contains data for about 1250 pharmaceuticals, including reference to about 400 hydrates among which about 200 have crystallographic data recorded. Guided by this information, and consulting the CSD via its online WebCSD search system (Thomas et al., 2010), we have located crystallographic data for 29 non-ionic pharmaceutical hydrate/parent systems. The systems thus identified are: acemetacin, acyclovir, ampicillin, aspartame, azathioprine, calcipotriol, carbamaze- pine, cholesterol, colchicine, cyclophosphamide, ephedrine, fluconazole, flurbiprofen, lactitol, mannitol, mercaptopurine, nevirapine, norfloxacin, olanzapine, paracetamol, phenobarbital, piracetam, praziquantel, prednisolone, rifampicin, sorbitol, sulfaguanidine, testosterone and zopiclone. Their molecular structures are depicted in Table S4 in the supporting information.

We here analyse these systems for their effective volumes of waters of crystallization, apart from aspartame and ephedrine for which the structural data are inconsistent.

\section{Results and discussion}

Table 1 is an example, extracted from Table S1, of our calculation of the effective volumes of waters of crystallization for a given pharmaceutical parent/hydrate system, in this case for the atypical antipsychotic olanzapine (Wikipedia, 2019). The third last column, headed $V_{\mathrm{m}}\left(\AA^{3}\right)$, lists the formula volumes of the materials in the corresponding rows as obtained from their CSD refcode data files (that is, cell volumes per formula unit $\left.V_{\text {cell }} / Z\right)$. Values in italics are regarded as outliers and disregarded in further analysis. In the penultimate column, headed $V\left(\mathrm{H}_{2} \mathrm{O}\right)\left(\AA^{3}\right)$, the value in bold is the mean of the formula unit volumes of the listed set of anhydrous parent molecules. Following beneath are the effective volumes of the waters of crystallization of the hydrates in the corresponding rows, calculated as the difference per water molecule between the volume of the hydrate and the mean volume of the parent:

$$
P\left(\text { parent } m \mathrm{H}_{2} \mathrm{O}\right)-P(\text { parent })=m \theta_{P, \mathrm{H} 2 \mathrm{O}} \text {. }
$$

The mean of these values (excluding outliers in italics) is listed at the base of the final column. It should be noted that effective volumes could also be calculated from the difference between hydrate pairs, $m$ and $n$ [following equation (1)], but this introduces a problem when one of a pair is incompatible 
with the other - and it is then unclear which of the two is at fault, whereas they are compared on the same basis when the parent is used as the fundamental reference.

Comment on outlier identification. As noted above, it is generally accepted that atoms and their combinations in molecules are incompressible under ambient conditions. If the introduced water can fit into gaps within the packed structure when a material becomes hydrated, then the effective volume will be less than the $30 \AA^{3}$ of liquid water - and this is the common observation. If the water can form a full set of four hydrogen bonds to its surroundings (an unlikely circumstance) then the effective volume will be similar to that of hexagonal ice, namely $32 \AA^{3}$. Finally, the parent molecule may undergo a polymorphic change in the process of hydration. Judging from Table S1, pharmaceutical polymorphic transitions generally involve a volume change of about $5 \AA^{3}$ (although acemetacin does exhibit a $10 \AA^{3}$ volume change). This suggests that the effective water volume may, in such unusual circumstances, reach up towards $40 \AA^{3}$. Thus, we will regard effective volumes up to about $33 \AA^{3}$ with some suspicion, and even larger values as outliers.

As an example, consider the data for nevirapine (Table S1). According to the CSD, the nevirapine hydrates TISJAH and TISJAH01 are hemihydrates, which leads to effective hydration water volumes of 60 and $40 \AA^{3}$, respectively. We class these as outliers, and suggest that they may possibly be monohydrates, unless the volume of the parent PABHIJ is seriously at issue; the Hofmann (2002) volume estimate (Table S2) suggests that this is indeed a possibility.

Fig. 1 provides an overview of the behaviour of parent volumes for polymorphs with varying temperature for paracetamol, with 47 crystal structures recorded (CSD) among three polymorphs and for temperatures ranging from 20 to $360 \mathrm{~K}$ (none of the data in the other hydrate/parent pairs are

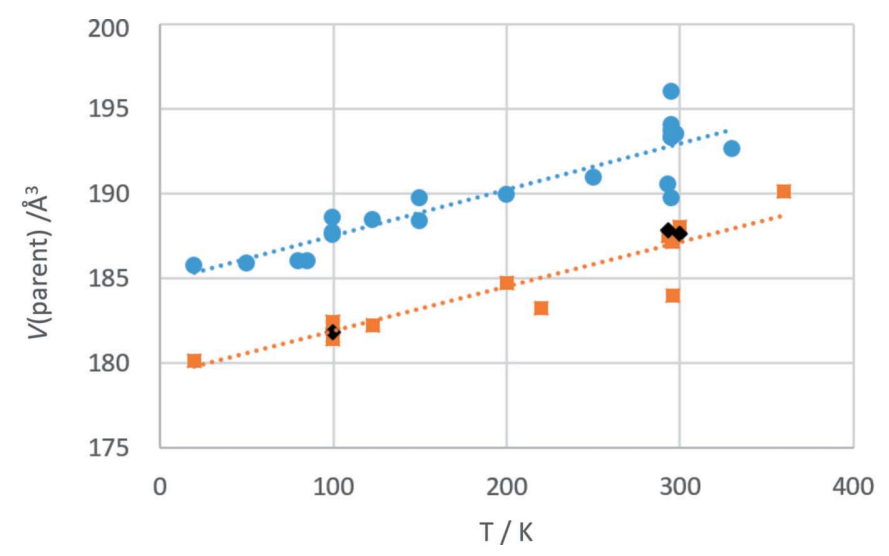

Figure 1

Formula volumes of paracetamol from 42 entries in the CSD, which include extensive data on thermal behaviour by Drebushchak \& Boldyreva (2004) and by Wilson (2000). Blue circles: polymorph I (monoclinic); orange squares: polymorph II (orthorhombic); black diamonds: polymorph III (orthorhombic). Polymorph I trendline: $V$ (parent) $\left(\AA^{3}\right)=0.0272 T+184.8$; polymorph II trendline: $V$ (parent) $\left(\AA^{3}\right)=0.0262 T+179.3$. Data in Table S3. A further five datum points for polymorph I at $295 \mathrm{~K}$ have been omitted as outliers since they are incompatible with the remaining data. sufficiently extensive to yield comparable figures). The corresponding data are listed in Table S2.

Fig. 2 depicts the frequency distribution of effective volumes of waters of crystallization for the set of materials in the supporting information file. Volumes greater than $30 \AA^{3}$ are regarded as excluded outliers because they are greater than the volume of liquid water, in common with the earlier observation of a firm upper limit in ionic hydrate/parent systems (Glasser, 2019) to the effective water volume.

Mention must be made of a few issues with regard to the materials listings in the supporting information file. (i) For a number of the more common materials, specifically carbamazepine, cholesterol, mannitol, paracetamol, phenobarbital and piracetam, there are multiple data files in the CSD with only representative example files listed. However the extensive parent data for paracetamol are plotted in Fig. 1 and listed in Table S3. (ii) For testosterone, the data for refcode TESTOM03 were initially listed as anhydrous but they are clearly for a monohydrate (by comparison with the remaining data) and the entry has now been corrected to a monohydrate. (iii) For nevirapine, the hydrates are listed as hemihydrates; the data suggest substantial errors perhaps in the parent volume, as noted above. (iv) The data for aspartame and for ephedrine are inconsistent, and have been omitted from our analysis.

\section{Conclusions}

We have collected data for 29 parent/hydrate non-ionic pharmaceutical pairs and analysed 27 of them for the effective volumes of their waters of crystallization, yielding a median volume of $22.8 \AA^{3}$. This corresponds well with the median value of $24.2 \AA^{3}$ for ionic parent/hydrate pairs. These values may be used in prediction of thermodynamic parameters, and are sufficiently reliable that it is possible to suggest where the published experimental data may be faulty.

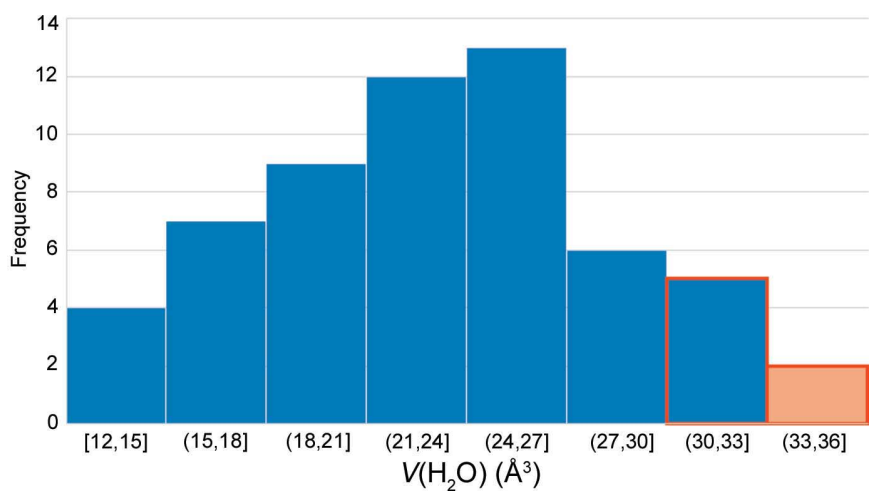

Figure 2

Frequency distribution of the effective volume per water of crystallization, $V\left(\mathrm{H}_{2} \mathrm{O}\right)\left(\AA^{3}\right)$, for 55 non-ionic pharmaceutical hydrate/parent pairs, having a median volume of $22.8 \AA^{3}$, with maximum 29.9 (3) $\AA^{3}$, minimum $11.7 \AA^{3}$ and mean $21.5 \pm 5.0 \AA^{3}$. The rightmost two columns represent seven possibly outlier data points excluded from the statistical analysis, with volumes above $30 \AA^{3}$. Data in Table S1. The data represented in the final column are here regarded as clear outliers while those in the preceding column are regarded with suspicion. 


\section{Acknowledgements}

The assistance of Dr E. Joseph Billo in adapting his Excel file for calculating molecular weights (Billo, 2011) to molecular volumes using data from Hofmann (2002) is gratefully acknowledged.

\section{References}

Billo, E. J. (2011). Excel for Chemists, 3rd ed. Hoboken, New Jersey: Wiley.

Drebushchak, T. N. \& Boldyreva, E. V. (2004). Z. Kristallogr. Cryst. Mater. 219, 506-512.

Fucke, K., Steed, J. \& Anderson, K. (2019). Hydrates database. http:// www.hydrateweb.org/database (accessed January 2019).

Glasser, L. (2014). J. Chem. Eng. Data, 59, 526-530.

Glasser, L. (2019). Cryst. Growth Des. 19, 3397-3401.

Glasser, L. \& Jenkins, H. D. B. (2004). Thermochim. Acta, 414, 125130.
Glasser, L. \& Jenkins, H. D. B. (2007). Inorg. Chem. 46, 97689778.

Glasser, L. \& Jenkins, H. D. B. (2016). Phys. Chem. Chem. Phys. 18, 21226-21240.

Glasser, L. \& Jones, F. (2009). Inorg. Chem. 48, 1661-1665.

Groom, C. R., Bruno, I. J., Lightfoot, M. P. \& Ward, S. C. (2016). Acta Cryst. B72, 171-179.

Hofmann, D. W. M. (2002). Acta Cryst. B58, 489-493.

Jenkins, H. D. B. \& Glasser, L. (2003). Inorg. Chem. 42, 87028708.

Jenkins, H. D. B. \& Glasser, L. (2004). J. Am. Chem. Soc. 126, 1580915817.

Jenkins, H. D. B. \& Liebman, J. F. (2009). J. Chem. Eng. Data, 54, 351358.

Thomas, I. R., Bruno, I. J., Cole, J. C., Macrae, C. F., Pidcock, E. \& Wood, P. A. (2010). J. Appl. Cryst. 43, 362-366.

Wikipedia (2019). Olanzapine. https://en.wikipedia.org/wiki/Olanzapine (accessed May 2019).

Wilson, C. C. (2000). Z. Kristallogr. Cryst. Mater. 215, 693-701. 